\title{
EDUCAÇÃO A DISTÂNCIA NA FORMAÇÃO EM ENFERMAGEM: REFLEXÕES SOBRE A PANDEMIA DA COVID-19
}

\section{DISTANCE LEARNING IN NURSING TRAINING: REFLECTIONS ON THE COVID-19 PANDEMIC}

\section{EDUCACIÓN A DISTANCIA EN LA ENSEÑANZA DE ENFERMERÍA: REFLEXIONES SOBRE LA PANDEMIA DE COVID-19}

\author{
Fabio Scorsolini-Comin ${ }^{1}$ \\ Lucas Pereira De Melo ${ }^{2}$ \\ Lucas Rossato ${ }^{3}$ \\ Ronan da Silva Parreira Gaia ${ }^{4}$
}

Como citar esse artigo: Scorsolini-Comin F, Melo LP, Rossato L, Gaia RSP. Educação a distância na formação em enfermagem: reflexões sobre a pandemia da COVID-19. Rev baiana enferm. 2020;34:e36929.

Objetivo: refletir sobre o emprego da educação a distância na graduação em enfermagem no Brasil no cenário da pandemia da COVID-19. Método: ensaio crítico por meio de reflexões ancoradas na literatura acerca da utilização da educação a distância na formação de enfermeiros(as) e dos circunscritores decorrentes da pandemia. Resultados: as discussões sobre o emprego da educação a distância na formação em enfermagem no Brasil respondem a diferentes interesses educacionais, profissionais, políticos e econômicos. No contexto da pandemia de COVID-19, a partir de 2020, tais debates têm sido potencializados em função do emprego de metodologias da educação a distância na continuidade de muitos cursos de formação, outrora exclusivamente presenciais. Conclusão: não obstante as metodologias próprias da educação a distância permitirem, em um primeiro momento, a continuidade dos processos formativos em enfermagem, reafirma-se que o ensino-aprendizagem para o cuidado em saúde demanda proximidade e contato.

Descritores: Educação a Distância. Educação em Enfermagem. Infecções por Coronavírus.

Objective: to reflect on the use of distance learning in nursing graduate programs in Brazil in the scenario of the COVID-19 pandemic. Method: critical essay through reflections anchored in the literature about the use of distance learning in the training of nurses and circumscriptors resulting from the pandemic. Results: discussions on the use of distance learning in mursing education in Brazil respond to different educational, professional, political and economic interests. In the context of the COVID-19 pandemic, since 2020, such debates have been intensified due to the use of distance learning methodologies in the continuity of many training courses, once exclusively in person. Conclusion: although the methodologies of distance learning allow, at first, the continuity of the training processes in nursing, it is reaffirmed that teaching-learning for health care demands proximity and contact.

Descriptors: Education, Distance. Education, Nursing. Coronavirus Infections.

\footnotetext{
Psicólogo. Mestre e Doutor em Psicologia. Docente do Departamento de Enfermagem Psiquiátrica e de Ciências Humanas da Escola de Enfermagem de Ribeirão Preto da Universidade de São Paulo. Ribeirão Preto, São Paulo, Brasil. https://orcid.org/0000-000 I-628 I-337। .

Enfermeiro. Mestre em Enfermagem. Doutor em Ciências. Docente do Departamento de Enfermagem Psiquiátrica e de Ciências Humanas da Escola de Enfermagem de Ribeirão Preto da Universidade de São Paulo. Ribeirão Preto, São Paulo, Brasil. https://orcid.org/0000-000 I-8392-1398.

3 Psicólogo. Mestre em Psicologia. Escola de Enfermagem de Ribeirão Preto da Universidade de São Paulo. Ribeirão Preto, São Paulo, Brasil. rossatousp@usp.br. https:// orcid.org/0000-0003-3350-0688.

4 Pedagogo. Escola de Enfermagem de Ribeirão Preto da Universidade de São Paulo. Ribeirão Preto, São Paulo, Brasil. https://orcid.org/0000-0003-2342-00।9.
} 
Objetivo: reflexionar sobre el uso de la educación a distancia en programas de graduación en enfermería en Brasil en el escenario de la pandemia COVID-19. Método: ensayo crítico a través de reflexiones ancladas en la literatura sobre el uso de la educación a distancia en la formación de enfermeras y circunscriptores resultantes de la pandemia. Resultados: los debates sobre el uso de la educación a distancia en la educación en enfermería en Brasil responden a diferentes intereses educativos, profesionales, politicos y económicos. En el contexto de la pandemia COVID-19, desde 2020, estos debates se han intensificado debido al uso de metodologias de educación a distancia en la continuidad de muchos cursos de formación, una vez exclusivamente presenciales. Conclusión: aunque las metodologías de la educación a distancia permiten, al principio, la continuidad de los procesos formativos en enfermerí, se reafirma que la enseñanza-aprendizaje para la atención de la salud exige proximidad y contacto.

Descriptores: Educación a Distancia. Educación en Enfermería. Infecciones por Coronavirus.

\section{Introdução}

No cenário da globalização atual, os processos educacionais são considerados como um dos seus pilares. Assim, a educação a distância (EaD) emerge não apenas como uma possibilidade, mas como uma realidade em muitos países, e sobretudo aplicada à formação de profissionais no ensino superior e na sua expansão. Essa modalidade de ensino pode ser aplicada por meio de diversos instrumentos ou mídias, sendo estes mediadores nos âmbitos da formação, treinamento, aprimoramento e atualização profissional.

A EaD emergiu em um cenário de avanços nas tecnologias de informação e comunicação, e a cada ano tem sido transformado e ampliado. Atualmente é considerada como importante estratégia para a educação em diferentes níveis de formação, inclusive no ensino superior, cenário no qual vem sendo alvo dos mais diversos embates, capitaneados por forças econômicas que defendem a sua instauração, e correntes educacionais que afirmam que, nem sempre, tal articulação é possível, sobretudo no contexto da formação para o cuidado em saúde ${ }^{(1)}$.

No Brasil, a expansão dos cursos de EaD ocorreu no final da década de 1980, e deu-se em consonância com a evolução das tecnologias educacionais e com eventos, como o maior acesso ao computador e à internet, elementos esses que vêm crescendo de modo intenso nos últimos anos ${ }^{(2)}$. A EaD tem despontado após a sua inclusão na Lei de Diretrizes e Bases da Educação Nacional, Lei Federal n. 9.394, de 20 de dezembro de $1996^{(3)}$. Com base nesse marco legal, diversas iniciativas passaram a ser desenvolvidas na tentativa de promover o ensino-aprendizagem, rompendo com paradigmas como a sincronia e a presença física de professores e estudantes em um mesmo espaço/tempo educacional. Obviamente que esse novo paradigma trouxe diversas flexibilizações, bem como demandou ajustes importantes, passando pelo ensino por correspondência, o ensino mediado pela TV e, mais recentemente, pelo advento da internet, dada sua popularização nos últimos $\operatorname{anos}^{(4)}$. Essas mudanças transformaram a EaD e, consequentemente, o modo de se pensar os processos educacionais nesse novo momento ${ }^{(5)}$.

Os objetivos das atividades de ensino-aprendizagem que se dão na modalidade a distância não se diferenciam daqueles apregoados na educação presencial, ou seja, o desenvolvimento e o aperfeiçoamento do aluno mediante diretrizes éticas, culturais e baseadas em um sólido paradigma formativo devem ocorrer independentemente da modalidade que se analisa. De igual monta, deve-se destacar que embora os currículos possam sofrer alterações, estes não podem ser reduzidos em função da modalidade, mas podem ser revistos e adaptados de acordo com marcadores, como nível de formação, público-alvo e características das tecnologias educacionais.

A EaD tem sido evidenciada como uma estratégia capaz de favorecer a aprendizagem contínua, devendo reconhecer a coletividade, a cooperação e a interação como marcadores do processo de ensino-aprendizagem ${ }^{(6)}$. Em uma perspectiva de aprendizagem colaborativa, isto 
é, baseada na interação e na cooperação, destaca-se que a EaD pode promover a horizontalização $^{(7)}$ das relações entre professor e aluno, de modo que o professor passa a ser um mediador da aprendizagem e não mais um detentor do saber. Em um ambiente virtual, esse profissional passa a ser o responsável por direcionar a aprendizagem do aluno, estabelecendo relações cooperativas e que favoreçam a adoção de estratégias de busca pelo conhecimento, de análise de evidências e de construção de uma atitude crítica diante desse processo formativo.

Ainda em relação ao papel do professor nesses cursos, destaca-se não apenas a necessidade do tutor mediar a aprendizagem, como também permitir o desenvolvimento contínuo da autonomia, da autoeficácia, da assertividade e da tomada de decisões ${ }^{(2)}$, competências fundamentais para a formação de alunos na contemporaneidade. Além disso, instituições que implementam essa forma de ensino devem promover condições para a troca de experiências entre docente-estudantes, estudantes-estudantes, informações e resolução de dúvidas que emergem durante o processo de aprendizagem. No caso dos cursos de formação em saúde, tais reflexões também devem incorporar aspectos como a necessidade de contato físico e presencial, como por exemplo, nos treinamentos em serviço ${ }^{(8)}$.

Em que pese a rápida expansão da $\mathrm{EaD}$ no Brasil, observa-se que poucas reflexões têm sido conduzidas para a adoção de uma postura mais crítica em relação à oferta de cursos de graduação e de pós-graduação nessa modalidade. Predominam, desse modo, relatos que asseveram a noção de flexibilidade como se este fosse o único elemento a ser perseguido em uma proposta formativa. A despeito dos demais marcadores da EaD, a flexibilidade muitas vezes emerge como uma condição que fragiliza o ensino, significada como elemento oposto à disciplina e ao controle dos processos educacionais. Tais posicionamentos podem refletir um desconhecimento em relação à EaD, o que conduzirá de modo inequívoco a discussão em torno do seu emprego na formação em saúde.
O cenário recente do novo coronavírus e da pandemia de COVID-19, a partir de 2020, tem reaquecido antigos debates, sobretudo quando consideramos a formação de profissionais de saúde exclusivamente por meio dessa modalidade. Neste ensaio, o foco se direciona aos cursos de graduação em enfermagem, uma vez que nesse campo as reflexões em torno da EaD têm sido mais proeminentes, ativando não apenas posicionamentos construídos com base em evidências científicas, mas também de entidades de classe, de instituições de ensino e demais audiências que respondem a diferentes objetivos e interesses ${ }^{(6-7,9)}$.

Assim, este ensaio tem por objetivo refletir sobre o emprego da EaD na graduação em enfermagem no Brasil no cenário da pandemia da COVID-19.

\section{Método}

No aspecto metodológico, este ensaio foi produzido após uma reflexão amparada na literatura científica acerca da formação de profissionais de enfermagem por meio dos pressupostos da $\operatorname{EaD}^{(9-10)}$. Esta reflexão propõe um debate baseado na literatura que possa agregar conhecimentos que se somem às produções em curso no cenário internacional que têm discutido a EaD na formação profissional em tempos da pandemia de COVID-19 $9^{(11-12)}$.

Nesse sentido, a exploração do contexto brasileiro pode ser de particular interesse, por representar movimentos distintos daqueles observados na produção internacional. Mais do que isso, a presente reflexão orienta-se para compreender que a pandemia é um evento situacional de grandes proporções, disparando a necessidade de problematizar suas repercussões especificamente para a formação em saúde. O ensaio reflexivo, portanto, parte do diálogo entre uma literatura já consolidada com um contexto ainda insipiente, em que evidências têm sido produzidas para subsidiar cada vez mais esse debate. 


\section{Resultados e Discussão}

\section{A EaD na formação em enfermagem no Brasil: notas para um debate}

No contexto nacional, o público da EaD tem se multiplicado nos últimos anos devido à flexibilização do regime de estudos que a modalidade oferece e aos preços mais acessíveis, sobretudo no ensino superior. O seu emprego também tem sido refletido como possibilidade de ampliar o acesso à educação a populações que vivem em regiões remotas, o que se mostra importante em um país marcado por desigualdades locorregionais como o $\mathrm{Brasil}^{(8)}$. No âmbito da política de saúde, destaca-se a Política Nacional de Educação Permanente em Saúde (PNEPS), que proporcionou ofertas educativas em EaD por meio da criação da Universidade Aberta do SUS (UNA-SUS), uma rede colaborativa de instituições de ensino, serviço e gestão do Sistema Único de Saúde (SUS) que tem possibilitado a capacitação de profissionais e técnicos ${ }^{(8)}$.

No Brasil, a enfermagem representa um campo que tem se mostrado fortemente receptivo a esse novo paradigma educacional, o que atinge não apenas os cursos de graduação como também os cursos de especialização, aprimoramento e capacitação profissional. Contudo, essa modalidade de ensino ainda é alvo de constantes debates das mais diversas ordens, com posicionamentos a favor e contrários à sua adoção nesse curso, em específico, pelo fato dessa profissão possuir, como uma das dimensões do trabalho, atividades gerenciais e de assistência à saúde, o que demanda contato presencial e interação humana nos contextos de ensino e de formação em serviço.

As evidências positivas que sustentam o emprego da EaD na formação em enfermagem destacam aspectos como a possibilidade de integração de mídias para simulação de cenários de prática, além da experimentação prática pelo aluno, sem que este coloque em risco a vida de um paciente real, apenas para citar dois exemplos. Além disso, tais atividades permitem ao aluno refletir sobre a tomada de decisões, mediante o processo de reunião de informações, levantamento de hipóteses, análise de contextos, bem como de riscos e benefícios ${ }^{(13)}$.

Essas estratégias de ensino-aprendizagem podem ser empregadas em cursos presenciais e mesmo em modalidades híbridas, de modo articulado ao ensino realizado face a face, ao treinamento em serviço e à efetivação de atividades que demandem o contato e a proximidade. O que se discute é que tais ferramentas, em um curso realizado a distância, recobririam todo o processo formativo, não havendo garantias de que as aprendizagens que demandam contato, presença e proximidade seriam de fato operacionalizadas.

As recomendações explicitadas na literatura ${ }^{(13)}$ consideram a EaD e a tele-enfermagem como possibilidades de atuação e mesmo de formação e atualização, de modo a constituírem estratégias empregadas de maneira complementar às metodologias consideradas "tradicionais", realizadas presencialmente. Assim, em um curso de graduação em enfermagem exclusivamente presencial, alguns conteúdos e disciplinas poderiam ser oferecidos empregando recursos próprios da $\mathrm{EaD}$, o que traria alguns benefícios aos estudantes, como o acesso a conteúdos mais recentes e o emprego de tecnologias inovadoras, o que poderia ser um incremento importante também considerando as metodologias ativas. Esse diálogo parece estar consolidado e reconhecido na literatura científica. Mas, e quando se pensa na oferta de um curso de enfermagem integralmente a distância?

No ensino de enfermagem torna-se um desafio utilizar essa modalidade na totalidade do curso, uma vez que ela coloca em suspensão o contato humano, ocorrido em situações presenciais, considerado fundamental ao ato de cuidar. No Brasil, o Conselho Federal de Enfermagem (COFEN) tem adotado posicionamentos contrários à abertura de cursos de graduação em enfermagem exclusivamente na modalidade $\mathrm{EaD}$, ressaltando que não é contra essa modalidade e suas estratégias de ensino-aprendizagem. Contudo, argumenta que as aulas práticas em 
laboratórios para o treinamento diário do estudante e a inserção na realidade dos serviços de saúde nos diferentes níveis de atenção do SUS, desde os anos iniciais do curso, são imprescindíveis para o aprendizado e para a boa formação do futuro profissional, pois the permite lidar com pessoas e problemas reais, os quais não são passíveis de recriação em ambientes virtuais ${ }^{(14)}$. Embora a simulação de cenários de prática tenha a sua importância, sobretudo para o estudante em início de curso, haja vista que essa aproximação pode diminuir a sua ansiedade diante do contato com o campo empírico, destaca-se que a ausência da experiência em campo, da interação presencial com o outro (colega, professor, paciente/cliente/usuário) possa afastar esse estudante do seu futuro contexto de atuação. As implicações para a formação da identidade profissional ainda precisam ser melhor investigadas na literatura científica, possivelmente comparando egressos de cursos presenciais com aqueles formados por meio da EaD em enfermagem.

A enfermagem, juntamente com outras profissões, tem empreendido esforços e problematizado a expansão da EaD em cursos da área da saúde, principalmente pela preocupação com a qualidade do ensino que é oferecido e pela dimensão prática que os cursos dessa área envolvem. Segundo o COFEN, a preocupação dos Conselhos em relação à $\mathrm{EaD}$ aumentou após o crescimento acelerado do número de cursos nessa modalidade nos últimos anos no Brasil. De 274.603 novas vagas autorizadas pelo Ministério da Educação (MEC), em 2017, o número saltou para 913.300 em 2019. Atualmente são reconhecidos 396 diferentes cursos de EaD no país ${ }^{(15)}$.

Ainda de acordo com o COFEN, as entidades representativas de enfermeiros também se posicionaram totalmente contrárias aos cursos de enfermagem integralmente em EaD, tanto em nível técnico quanto superior, pois acreditam que para a formação e o exercício dessa profissão é fundamental o contato humano, o que não pode ser substituído por qualquer tipo de tecnologia $^{(16)}$. Aventa-se, nesse contexto da hipermodernidade, que a inovação na formação em enfermagem não seja necessariamente o emprego de tecnologias capazes de facilitar o aprendizado ou simular os cenários de prática, mas justamente retomar e investir em uma formação próxima, humanizada e que pressupõe o contato presencial, a corporeidade.

Além dos Conselhos, associações e sociedades científicas e profissionais, o Conselho Nacional de Saúde (CNS) emitiu, em 2018, uma nota pública, para se posicionar de forma contrária a essa metodologia de ensino nos cursos que formam os profissionais da área. Assinada por 58 instituições, a nota entoa uma voz que merece ser escutada, pois expressa uma perspectiva daqueles que vivem a prática das atividades em saúde no Brasil. Segundo eles, a expansão das matrículas e/ou a necessidade social da EaD não justifica a adoção dessa modalidade de ensino para formar profissionais que atuam na área da saúde, isso porque sobram vagas nas modalidades presenciais.

A nota não aborda, entretanto, acerca da expansão do curso em áreas remotas, onde não existem instituições de ensino para formar profissionais da saúde, mas aponta que é preciso aprofundar o debate sobre as políticas públicas de educação, considerando a necessidade de cada curso. O CNS ressalta ainda que não se opõe às oportunidades que as tecnologias oferecem à formação dos cursos superiores da modalidade presencial e que reconhece a qualificação dos processos pedagógicos promovidas pelo desenvolvimento das ferramentas virtuais.

Cumpre ressaltar que os números aqui apresentados, e mesmo os posicionamentos desses órgãos, ganham dimensões importantes, se tomados contra o pano de fundo do modelo de expansão da educação superior brasileira, iniciado nos anos 2000, cuja tônica foi marcada pelo crescente predomínio dos interesses privados/ mercantis e pela flexibilização da regulação estatal, um processo denominado como "corrida do ouro' da educação-mercadoria e do mercado educacional" (17:1015, grifos do autor) . No âmbito curricular, problematiza-se as reverberações dessas lógicas neoliberais nos processos de ensino e aprendizagem, no caso, as metodologias 
ativas de ensino-aprendizagem, em suas diversas esferas, nomeadamente no que diz respeito a uma pedagogia que se volta cada vez mais ao indivíduo gestor de si e de sua aprendizagem, flexível, disponível a aprender, cujos conhecimentos estão ajustados às demandas econômicas do mercado ${ }^{(18)}$.

Dessa forma, não obstante ser um recurso cada vez mais presente nas práticas de ensino, percebe-se que a EaD mantém atrelada a si polêmicas em relação à sua constituição e à forma como se estabelece. Como já apontado, o problema não é a $\mathrm{EaD}$ per se, mas seu uso exclusivo e integral como modalidade de ensino em cursos da área da saúde. A EaD, em nosso país, foi historicamente associada a uma educação de menor qualidade justamente por ser, inicialmente, uma alternativa às pessoas que não podiam se engajar no ensino tradicional, quer seja por exigências laborais, quer seja por não estarem no período de escolarização típico ${ }^{(19)}$. Entretanto, hoje se conhece experiências exitosas em que a EaD foi empregada de forma efetiva e eficiente, bem planejada, antenada com os desenvolvimentos no campo das tecnologias de informação e comunicação e com arranjos curriculares que garantem a qualidade da formação.

A evolução da EaD ao longo dos anos e o aprimoramento de suas ferramentas educacionais após os avanços das tecnologias digitais de informação e comunicação também tem permitido maior interação entre os sujeitos da aprendizagem, favorecendo a troca, a mediação e a colaboração, permitindo um acompanhamento de maior qualidade ${ }^{(19)}$. Esses incrementos tecnológicos, fundamentais à melhoria da qualidade da formação por meio de EaD, também devem ser acompanhados com base na capacitação dos profissionais que atuam nesses cursos, ampliando as expertises com as quais os alunos podem entrar em contato.

Em contraposição aos aspectos positivos que foram se adensando com o passar do tempo, deve-se problematizar que a implementação desenfreada dessa modalidade de ensino, muitas vezes sem planejamento, com vistas a atender as necessidades de cada curso puramente com finalidade econômica das instituições de ensino, tem levantado um alerta para os Conselhos Profissionais em relação ao tipo de profissional que estará sendo formado e suas reais capacidades técnicas para lidar com os problemas cotidianos da prática em saúde. Desse modo, todos esses elementos acabam contribuindo para que a $\mathrm{EaD}$, na área da enfermagem, gere debates e problematizações que são importantes e devem serem considerados.

Se anteriormente a EaD apresentava-se fundamentalmente como uma estratégia complementar à educação tradicional e presencial, a expansão dessa modalidade no ensino superior privado tem possibilitado a oferta de diversos cursos da área de saúde integralmente a distância ${ }^{(17)}$. Tais ofertas muitas vezes não são acompanhadas de reflexões importantes ao fazer profissional, o que pode trazer, como consequência, riscos significativos à qualidade da atenção à saúde ${ }^{(20)}$.

Outro aspecto importante de ser evidenciado em relação à $\mathrm{EaD}$, refere-se aos altos índices de evasão de alunos nessa modalidade de ensino. A evasão pode ser evidenciada como um dos principais problemas da EaD, pois há casos em que apenas a metade dos estudantes concluíram os programas de formação. As principais causas para evasão são: falta de tempo; situação financeira; não adaptação ao ambiente virtual do curso; distância; não dedicação aos estudos; não terem recebido os recursos necessários da instituição; o curso não ser exatamente o que queriam; ausência de interação com outros estudantes, entre outros ${ }^{(21)}$.

Diante do cenário da pandemia de COVID-19 essas questões não se descaracterizam. Pelo contrário, podem ser potencializadas. Assim, é importante que haja a continuidade dessas investigações, tendo em vista as especificidades decorrentes da pandemia e também a velocidade com que essas mudanças são implementadas e também revistas. Em um cenário de impermanência, é importante que as questões que balizam a discussão sobre a possibilidade ou não de uma graduação em enfermagem totalmente na modalidade a distância possam refletir, não a necessidade de ajustamento à realidade da 
pandemia, mas sim, o cotejamento de uma realidade educacional e profissional nacional que, independentemente das transformações oportunizadas pela pandemia, devem, muito provavelmente, se manter. Isso ocorre quando consideramos aspectos como o acesso à educação, a valorização das profissões de enfermagem, o perfil dos alunos desses cursos, o valor pago pelos estudantes em cursos privados na modalidade $\mathrm{EaD}$, a remuneração profissional dessa categoria, entre diversos outros atravessamentos que formam o nosso contexto educacional.

Embora o ensino remoto esteja sendo considerado no contexto da pandemia como uma resposta urgente, buscando a manutenção dos processos de ensino e aprendizagem, sobretudo para a formação de profissionais que possam atuar na chamada "linha de frente de combate", caso no qual se enquadra a enfermagem, é importante considerar que esse cenário não pode significar, de modo automático, a exequibilidade de uma formação em enfermagem exclusivamente a distância. Embora a EaD esteja contribuindo no atual cenário com a oferta de ferramentas e metodologias que permitam a continuidade dos processos formativos em cursos presenciais, não se trata de afirmar que esses cursos passaram a ser ofertados na modalidade EaD. Os pressupostos que orientam esses cursos continuam sendo os do modelo presencial, ainda que o emprego de recursos da EaD esteja favorecendo - e também possibilitando - a manutenção da formação em um cenário no qual o distanciamento social tem se mostrado uma das únicas estratégias de contenção do contágio. As ações ofertadas na atualidade e as reflexões existentes no contexto da pandemia ainda são insipientes, mas retratam sobretudo as potencialidades da EaD para o ensino remoto, não em uma estratégia de substituição ao ensino presencial, mas justamente de não interrupção dos processos formativos ${ }^{(9-10)}$.

O que tem sido observado nos cursos presenciais de enfermagem que têm adotado estratégias de ensino-aprendizagem inspiradas na EaD é que a formação teórica tem sido possível com a utilização desses recursos. A formação em serviço e a realização de atividades laboratoriais não têm sido operacionalizadas a distância, pelo contrário, têm sido adiadas e replanejadas em função da evolução da pandemia em nosso contexto. Assim, a EaD tem contribuído com parte dessa manutenção, mas obviamente não substitui aquilo que se compreende como essencial na formação para o cuidado em enfermagem, isto é, a presença, a corporeidade, a proximidade. Em um cenário de instabilidade, essas reflexões deverão ser acompanhadas ao longo da pandemia e da possível retomada das atividades presenciais no futuro.

Desse modo, a pandemia pode ser um elemento importante para reaquecer o debate acerca da formação em enfermagem a distância, mediante o fornecimento de mais evidências que possam, futuramente, subsidiar o planejamento desse modelo formativo. No entanto, assevera-se que não se trata apenas de uma produção de evidências, mas que elas possam ser refletidas criticamente para uma educação possível, exequível e que possa ser oferecida aos estudantes, futuros enfermeiros, e aos profissionais que efetivamente pretendemos formar, não apenas para um mercado de trabalho com fortes demandas, mas para uma sociedade na qual a saúde não seja uma mercadoria, mas um direito e uma responsabilidade de todos.

\section{Conclusão}

Tendo em vista o argumento desenvolvido neste ensaio, considera-se a importância de que a EaD, na formação em enfermagem, possa ser melhor discutida e não apenas no cenário da pandemia de COVID-19. Para além das inequívocas contribuições das estratégias de ensino-aprendizagem, a distância surge como adaptação a um contexto de isolamento e distanciamento social que não tem possibilitado a manutenção do ensino presencial nas universidades brasileiras. Reforça-se que a implementação de cursos de enfermagem integralmente a distância constituem um debate antigo, que não pode ser 
enviesado em função de novos circunscritores que ainda não se pode estimar a sua amplitude nem mesmo a sua duração.

Em um cenário de instabilidade, com uma pandemia em trânsito e com repercussões ainda desconhecidas no contexto brasileiro, o que pode ter implicações diretas para a formação em saúde, considera-se que essa discussão em torno da formação em enfermagem via EaD deve ser conduzida com prudência, primando por um exercício que possa ser conduzido independentemente de contextos políticos e econômicos que possivelmente estejam aviltados em decorrência da pandemia em curso.

Os conselhos de classe devem acompanhar esse cenário, reafirmando seus posicionamentos em defesa de um ensino de qualidade que permita uma adequada formação para o cuidar. Essa formação, pelos argumentos aqui expostos, embora possa se beneficiar de estratégias inovadoras capitaneadas pela $\mathrm{EaD}$, não pode prescindir de elementos essenciais no cuidado, como a presença, o contato, a corporeidade e a interação face a face que, histórica e culturalmente, compõem o nosso fazer em enfermagem.

\section{Fonte de Financiamento:}

Coordenação de Aperfeiçoamento de Pessoal de Nível Superior (CAPES).

\section{Colaborações:}

1 - concepção, projeto, análise e interpretação dos dados: Fabio Scorsolini-Comin, Lucas Pereira de Melo, Lucas Rossato e Ronan da Silva Parreira Gaia;

2 - redação do artigo e revisão crítica relevante do conteúdo intelectual: Fabio Scorsolini-Comin, Lucas Pereira de Melo, Lucas Rossato e Ronan da Silva Parreira Gaia;

3 - aprovação final da versão a ser publicada: Fabio Scorsolini-Comin, Lucas Pereira de Melo, Lucas Rossato e Ronan da Silva Parreira Gaia.

\section{Referências}

1. Freire LM, Paula MA, Duarte ED, Bueno M. Educação a distância no cenário da enfermagem neonatal: revisão sistemática. Rev Esc Enferm USP. 2015;49(3):515-21. DOI: 10.1590/S0080-62342015 0000300021

2. Marin MJS, Nascimento EN, Tonhom SFR, Alves SBAD, Girotto MA, Otani MAP, et al. Formação na modalidade a distância pela Universidade Aberta do SUS: Estudo qualitativo sobre o impacto do curso na prática profissional. Rev bras educ méd. 2017;41(2):201-9. DOI: http://dx.doi.org/ 10.1590/1981-52712015v41n2RB20160003

3. Oliveira MAN. Educação à distância como estratégia para a educação permanente em saúde: possibilidades e desafios. Rev bras enferm. 2007;60(5):585-9. DOI: 10.1590/S003471672007000500019

4. Alves VLS, Bohomol E, Cunha ICK. Educação de pós-graduação em enfermagem à distância: avaliação sob a perspectiva dos discentes. Acta paul enferm. 2015;28(2):139-45. DOI: 10.1590/ 1982-0194201500024

5. Scorsolini-Comin F. Diálogo e dialogismo em Mikhail Bakhtin e Paulo Freire: contribuições para a educação a distância. Educ rev. 2014;30(3):245-65. DOI: $10.1590 /$ S0102-46982014000300011

6. Paulon SM, Carneiro MLF. A educação a distância como dispositivo de fomento às redes de cuidado em saúde. Interface comun saúde educ. 2009;13(1):749-57. DOI: 10.1590/ S1414-32832009000500026

7. Vargas FMA, Gouveia GDA, Trindade MCN, Farias MR. A educação a distância na qualificação de profissionais para o Sistema Único De Saúde: metaestudo. Trab educ saúde. 2016;14(3):849-70. DOI: 10.1590/1981-7746-sol00018

8. Cezar DM, Paz AA, Costa MR, Pinto MEB, Magalhães CR. Percepções dos médicos sobre a educação a distância e a contribuição da especialização em saúde da família. Interface (Botucatu). 2019;23(Suppl 1):e180037. DOI: http:// dx.doi.org/10.1590/interface.180037

9. Bezerra IMP. Estado da arte sobre o ensino de enfermagem e os desafios do uso de tecnologias remotas em época de pandemia do corona vírus. Rev bras crescimento desenvolv hum. 2020;30(1):141-7. DOI: 10.7322/jhgd.v30.10087 
10. Jowseya T, Fosterb G, Cooper-Ioeluc P, Jacobs S. Blended learning via distance in pre-registration nursing education: a scoping review. Nurse Educ Pract. 2020;44:102775. DOI: 10.1016/j.nepr. 2020.102775

11. Chick RC, Clifton GT, Peace KM, Propper BW, Hale DF, Alseidi AA, et al. Using technology to maintain the education of residents during the COVID-19 pandemic. J Surg Educ. 2020;77(4):729-32. DOI: $10.1016 /$ j.jsurg.2020.03.018

12. Warhadpande S, Khaja MS, Sabri SS. The impact of COVID-19 on interventional radiology training programs: what you need to know. Acad Radiol. 2020 Jun;27(6):868-71. DOI: 10.1016/j.acra.2020. 04.024

13. Rodrigues RCV, Peres HHC. Panorama brasileiro do ensino de Enfermagem on-line. Rev Esc Enferm USP. 2008;42(2):298-304. DOI: 10.1590/ S0080-62342008000200013

14. Conselho Federal de Enfermagem. 4 perguntas sobre o Cofen e o EaD na Enfermagem [Internet]. Brasília(DF); 2017 [cited 2020 Jun 4]. Available from: http://www.cofen.gov.br/4-perguntas-sobreo-cofen-e-o-ead-na-enfermagem_48429.html

15. Conselho Federal de Enfermagem. Conselhos lutam contra o EaD nos cursos de saúde. MEC defende modelo híbrido [Internet]. Brasília (DF); 2019 [cited 2020 Jun 7]. Available from: http:// www.cofen.gov.br/conselhos-lutam-contra-oead-nos-cursos-de-saude-mec-defende-modelohibrido_73655.html

16. Conselho Federal de Enfermagem. Enfermeiros são contra cursos a distância de Enfermagem [Internet].
Brasília (DF); 2018 [cited 2020 Jun 14]. Available from: http://www.cofen.gov.br/enfermeiros-saocontra-cursos-a-distancia-de-enfermagem_62939. html

17. Sguissardi V. Modelo de expansão da educação superior no Brasil: predomínio privado/mercantil e desafios para a regulação e a formação universitária. Educ Soc. 2008;29(105):991-1022. DOI:https://doi.org/10.1590/S0101-73302008000400004

18. Silva RRD. Estetização pedagógica, aprendizagens ativas e práticas curriculares no Brasil. Educ Realidade. 2018;43(2):551-68. DOI: 10.1590/2175623667743

19. Ortiz MCL, Ribeiro RP, Garanhani ML. Educação à distância: uma ferramenta para educação permanente de enfermeiros que trabalham com assistência perioperatória. Cogitare enferm. 2008;13(4):558-65. DOI: http://dx.doi.org/10.5380/ ce.v13i4.13116

20. Machado MH, Ximenes Neto FRG. Gestão da educação e do trabalho em saúde no SUS: trinta anos de avanços e desafios. Ciênc Saúde Colet. 2018;23(6):1971-9. DOI: 10.1590/ $1413-81232018236.06682018$

21. Abbad GS, Zerbini T, Souza DBL. Panorama das pesquisas em educação a distância no Brasil. Estud psicol. 2010;15(3):291-8. DOI: 10.1590/ S1413-294X2010000300009

Recebido: 22 de maio de 2020

Aprovado: 1 de julho de 2020

Publicado: 24 de agosto de 2020

A Revista Baiana de Enfermagem utiliza a Licença Creative Commons - Atribuição-NãoComercial 4.0 Internacional. https://creativecommons.org/licenses/by-nc/4.0/

Este artigo é de acesso aberto distribuído sob os termos da Licença Creative Commons (CC BY-NC). Esta licença permite que outros remixem, adaptem e criem a partir do seu trabalho para fins não comerciais. Embora os novos trabalhos tenham de lhe atribuir o devido crédito e não possam ser usados para fins comerciais, os usuários não têm de licenciar esses trabalhos derivados sob os mesmos termos. 